frequency was more than once a week in $52 \%$, once a week in $21 \%$, and once a month in $27 \%$. Intensity was severe in $30 \%$, and mild in $40 \%$. Localization was frontal in $69 \%$ and occipital in 23\%; bilateral in $48 \%$ and alternating in $22 \%$. Headache associated symptoms in $47 \%$ included photophobia (15\%), nausea (7\%), and vertigo (8\%). A psychogenic precipitant was recognized in $22 \%$. A history of periodic syndrome, mainly cyclic vomiting and recurrent abdominal pain, preceded the onset of headache illness in $47 \%$. Family history of migraine was present in $58 \%$. Only $14 \%$ of patients had other types of headache in addition; $10 \%$ had migraine and $4 \%$ tension headache. Neurologic exam, imaging in 32 patients, and EEG in 67 were normal. At 1 to 5 year followup, $70 \%$ were free of symptoms. (Soriani S, Battistella PA, Arnaldi C et al. Juvenile idiopathic stabbing headache. Headache Oct 1996;36:565-567). (Respond: Dr S Soriani, Paediatric Institute, Ferrara Univ, via Savonarola 9, 44100 Ferrara, Italy).

COMMENT. A small group of juvenile headache patients with characteristically very brief attacks of stabbing pain, with onset around 7 years, and spontaneous remission usually within 1 to 5 years, may deserve greater recognition as a childhood headache syndrome with a relatively favorable prognosis. A previous report cited by the authors found a $25 \%$ incidence of EEG abnormalities among patients with this syndrome. (Kramer JW et al. The value of the EEG in children with chronic headache. Brain Dev 1994;16:304-308). Others have shown a high incidence of EEG abnormalities in migraine patients and a beneficial response to the anticonvulsant, phenytoin. (Millichap JG. Child's Brain 1978;4:95-105). The use of valproate in headache patients is reviewed in the following article.

\title{
DIVALPROEX SODIUM IN HEADACHE
}

An approach to treating migraine with the anticonvulsant, divalproex sodium, is reviewed from the Comprehensive Headache Center, Germantown Hospital, Philadelphia, PA. Four double-blind, placebo-controlled studies have confirmed the efficacy of valproate in treatment of migraine. The frequency of attacks as well as the duration and intensity were reduced. The most frequent adverse effects included nausea, asthenia, dyspepsia, dizziness, somnolence, and diarrhea. The use of valproate for headache prevention in children under 10 years should be avoided, except in exceptional cases. (Silberstein SD. Divalproex sodium in headache: literature review and clinical guidelines. Headache Oct 1996;36:547-555). (Respond: Dr Stephen D Silberstein, Germantown Hospital, One Penn Boulevard, Philadelphia, PA 19144).

COMMENT. The occurrence of liver toxicity in children treated with valproate, between 1:500 to 1:9000, prompts caution since the reaction may be fatal. Attention to possible dietary factors in the cause of migraine, and the initiation of less toxic medications or alternative treatments should be investigated thoroughly before resorting to valproate therapy.

The acute treatment of migraine with Rizatriptan vs Sumatriptan has been studied in 10 US and 4 Dutch investigator centers, involving 449 patients, and is reported from the Department of Neurology, Leiden University Hospital, the Netherlands. (Visser WH, Ferrari MD et al. Arch Neurol Nov 1996;53:1132-1137). The antimigraine effect of 10 and $20 \mathrm{mg}$ rizatriptan was superior to placebo, and equal to $100 \mathrm{mg}$ sumatriptan succinate; $40 \mathrm{mg}$ rizatriptan was superior to $100 \mathrm{mg}$ sumatriptan succinate in efficacy but caused frequent side effects. 